\title{
63 Ausdauersport und Fettgewebe
}

\author{
(c) Springer-Verlag GmbH Deutschland, ein Teil von Springer Nature 2018 \\ D. Mathias, Fit und gesund von 1 bis Hundert \\ https://doi.org/10.1007/978-3-662-56307-6_63
}

Wichtige positive Effekte des Sports sind:

- Abbau von überflüssigem Fettgewebe mit deutlich positiven Auswirkungen auf den Stoffwechsel und das Herz-Kreislauf-System

- Senkung der Rate chronischer Erkrankungen

- Allgemeine Reduktion des Körpergewichts und damit neben dem psychischen Wohlbefinden auch Entlastung der Gelenke

Der gesunde Körperfettanteil beträgt bei jungen, normalgewichtigen Frauen etwa 25\%, 30 Jahre später sind es rund $5 \%$ mehr. Bei Männern betragen diese Werte ca. $18 \%$ und $25 \%$. Der Körperfettanteil hängt dabei nicht nur vom Geschlecht, Alter und der Ernährung ab, sondern wird entscheidend auch vom Trainingszustand geprägt. Erhöhte Fettanteile sinken durch regelmäßige körperliche Aktivitäten deutlich. Bei diesem Abbau von Fettgewebe sind in erster Linie die Fettpolster am Bauch betroffen. Das ist positiv, weil Fettgewebe hier besonders gesundheitsschädlich ist ( $\triangleright$ Kap. 42 und $\triangleright$ Kap. 44). Auch die Fettsäurespiegel im Blut sinken.

Nur wenig verändern sich durch Sport die Cholesterinwerte. Die Daten vieler randomisierter, kontrollierter Studien zeigen ein geringes Ansteigen der Konzentrationen des HDL-Cholesterins und ein leichtes Sinken der Spiegel des LDL-Cholesterins. Die LDL-Partikel nehmen durch Ausdauersport in ihrer Größe etwas zu, was zur Verminderung des Arterioskleroserisikos beiträgt, weil kleinere LDL-Partikel risikoreicher sind ( $\$ Kap. 18).

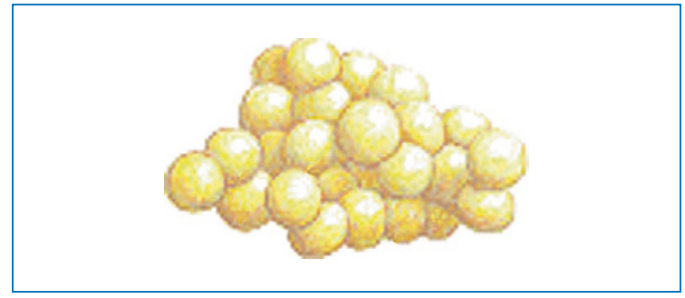

- Abb. 63.1

Auf Übergewicht und Fettleibigkeit ist ein Großteil aller Gelenkersatzoperationen an Hüfte und Knie zurückzuführen (Anandacoomarasamy et al. 2009; Wang et al. 2009; Smith et al. 2014b). Verantwortlich für die ursächlichen Gelenkschäden sind nicht nur die überflüssigen Pfunde, die getragen werden müssen, sondern auch das vom Fettgewebe gebildete Hormon Leptin ( $\downarrow$ Kap. 13). Dieses kann die Gelenkknorpel über eine schleichende Entzündungsreaktion zerstören. Der Abbau von Fettgewebe durch Ausdauersport wirkt deshalb auf die Gelenke doppelt positiv. Es lastet dann ein niedrigeres Gewicht auf ihnen und weniger Leptin entfaltet seine schädliche Wirkung.

Nicht zu unterschätzen ist der ästhetische Gesichtspunkt. Die durch mehr Bewegung bedingte Reduzierung des Bauchumfangs stärkt meist erheblich das Selbstwertgefühl. 